Running head: A GRICEAN APPROACH TO COVARIATON-BASED

\title{
ATTRIBUTION
}

NOTICE. This is the accepted version of a paper that has appeared as Maris, S., \& Hoorens, V. (2014). A Gricean approach to the use of consensus and distinctiveness in causal attributions of verbally described interpersonal events. European Journal of Social Psychology, 44, 141-153. It may not exactly replicate the published version. It is not the copy of record.

A Gricean Approach to the Use Of Consensus And Distinctiveness in Causal Attributions of Verbally Described Interpersonal Events

$$
\text { Stefanie Maris }{ }^{1,2} \text { and Vera Hoorens }{ }^{2}
$$

FWO-Flanders ${ }^{1}$, University of Leuven ${ }^{2}$, Leuven, Belgium

Author Note

Stefanie Maris, aspirant Fonds voor Wetenschappelijk Onderzoek Vlaanderen.

Stefanie Maris and Vera Hoorens, Center for Social and Cultural Psychology, University of Leuven, Belgium.

The authors thank John Skowronski, Denis Hilton, and several anonymous reviewers for their comments on an earlier draft and/or on conference presentations about (parts of) this paper. Correspondence concerning this article should be addressed to Vera Hoorens, Center for Social and Cultural Psychology, University of Leuven, Tiensestraat 102 box 3727, 3000 Leuven, Belgium. E-mail: Vera.Hoorens@ppw.kuleuven.be. 


\begin{abstract}
Various causal attribution theories, starting with the covariation model, argue that people use consensus, distinctiveness, and consistency information to causally explain events and behaviors. Yet the visual presentation of the covariation model in the form of a cube is based on the assumptions that these dimension generally affect attributions independently, symmetrically, and to equal extents. A Gricean analysis suggests that these assumptions may not generally hold in the case of causal judgments for verbally communicated interpersonal events. We had participants judge the causal role of an actor and a patient in interpersonal events that were described through actor-verbpatient sentences under high versus low consensus and distinctiveness (Study 1-2-3) or without such information (Study 2-3). As predicted by Gricean logic, consensus and distinctiveness effects on causality ratings depended on the target whose causal role participants assessed, on the information about the alternative dimension, and, most consistently, on consensus and distinctiveness being high versus low.
\end{abstract}

Key words: Causal attribution, covariation model, logic of conversations, consensus information, distinctiveness information 
A Gricean Approach to the Use Of Consensus And Distinctiveness in Causal Attributions of Verbally Described Interpersonal Events

Why did Peter kiss Mary? Why did Mary invite Peter? People often wonder why interpersonal events occur. In many cases, they base their attributions on limited information. For instance, people often know of the events and about these events' contexts through what others tell them. As causal attributions subsequently affect how observers respond to those involved, it is important to understand how these observers answer attribution questions. We therefore examined how people pass causal judgments about interpersonal events they know of through hearsay.

One influential model of causal attributions is Kelley's covariation model (Kelley, 1967, 1973). It was not specifically developed to explain attributions of interpersonal events, let alone interpersonal events about which people are solely informed through verbal descriptions. Yet, empirical tests have included a mixture of social and nonsocial events and most have used verbal behavioral descriptions as stimulus materials. It seems, then, that users of the covariation model assume that attributions of social and nonsocial events obey the same rules and that verbal descriptions of behaviors are functionally equivalent to directly observed behaviors.

At least three more specific assumptions implicitly underlie the covariation model. These include the ideas that people use information about different covariation dimensions to a similar extent, that they consider information on a dimension independently from the information on other dimensions, and that they weigh high and low levels equally. In the case of verbally described interpersonal events, however, an application of Grice (1975)'s analysis of human conversation suggests that these assumptions may not always hold. We therefore examined the validity of the 
assumptions underlying the covariation model by testing hypotheses derived from a Gricean approach. More specifically, we tested an attribution question hypothesis (stating that the use of consensus and distinctiveness depends on whose causal role people assess), an interdependence hypothesis (stating that the use of consensus and distinctiveness depends on information about the alternative dimension), and an asymmetry hypothesis (stating that high versus low consensus and distinctiveness affect causal judgments to different extents). Before explaining how and why the covariation model challenges the assumptions of the covariation model, however, we will briefly describe the model.

\section{The covariation model}

The covariation model, also known as the cube model, states that people arrive at causal attributions by scrutinizing, besides information about the to-be-explained event itself, different types of contextual information. People examine how the to-beexplained events covary with actors, patients, and subsequent occurrences, and derive causal attributions from the observed covariation. Stated differently, the model states that people consider contextual information on the dimensions of consensus, distinctiveness, and consistency.

Consensus is about the uniqueness of the actor's behavior. It is high if many people behave similarly (e.g. many people kiss Mary) and low if few people behave similarly. Distinctiveness is about the uniqueness of the patient's treatment. It is high if few people are similarly treated (e.g. Nobody else gets kissed) and low if many people are similarly treated. Consistency is about how uniformly the event occurs over times and circumstances. It is high if the event occurs at several times and in various situations (e.g. Peter often kisses Mary) and low if it happens at specific times or in specific 
circumstances only. Actor attributions occur if consensus and distinctiveness are low and consistency is high. Patient attributions occur if consensus, distinctiveness, and consistency are high. Other combinations provoke attributions to situational factors or to interactions between the actor, the patient, and the situation.

The covariation model does not detail how people weigh covariation information. Yet, the absence of any specification of factors affecting the use of the dimensions as well as the model's graphical presentation as a perfect cube suggest that people use each type of dimension to a similar extent and that they treat information about high and low positions in a symmetrical manner. Finally, people are assumed to collect and use information about any given dimension regardless of the information they have already available about any other dimension. If, for instance, an observer happens to first notice that distinctiveness is high, then this observation is supposedly irrelevant for the extent to which that observer subsequently searches and uses consensus information. It is only after all available covariation information is gathered that the covariation pattern is determined and the dimensions of covariation together determine the observer's causal conclusion.

Tests of the covariation model have yielded inconsistent results. Many studies showed that people can and sometimes do apply the covariation principle to the dimensions identified by the model and as the model predicts (e.g. Iacobucci \& McGill, 1990). Yet, other studies revealed that even when applying the covariation principle people do not always consider any information on any dimension equally important, nor uniformly use it. One explanation for the inconsistency is that different studies focused on different aspects of the attribution process. Preferences concerning the acquisition of information or concerning the normative judgment of information may well be 
dissociated from preferences concerning actual information use (Major, 1980). It should therefore not come as a surprise that different measures yield inconsistent results. Yet, we submit that the inconsistency is also, and perhaps mainly, due to a neglect of the extent to which the conversational context of the experimental communication about stimulus events and attribution questions affect participants' actual use of covariation information towards explaining interpersonal events.

\section{Testing the covariation model: A Gricean view}

Previous research has often treated the verbally described to-be-explained events and the attribution questions that participants are presented with in attribution studies as occurring within a social vacuum. To fully understand attribution processes occurring in the context of verbally communicated events, however, it is advisable to view the experimental communication within its broader conversational context. In this section, we explain the predictions that a Gricean analysis inspires about how people use different types of covariation information in causal judgments.

Before continuing, it is important to note that while trying to explain interpersonal events people above all wish to know which interactant is most responsible for the event. Consensus and distinctiveness differentiate between actor and patient attributions whereas consistency does not. Hence, to test predictions of the covariation model as it applies to interpersonal events it is most useful to examine effects of consensus and distinctiveness. Our application of a Gricean analysis to covariation-based attribution therefore focuses on the use of distinctiveness and consensus information.

Grice (1975)'s analysis states that conversations are governed by the assumption that communication partners follow a principle of cooperation, which makes them follow a set of basic rules ('maxims'). One is the 'maxim of relation', which implies 
that any message should be relevant for the goals of the conversation. This maxim implies that communications about an actor doing something to a patient suggest that this information is worth sharing. Previous research has shown that Grice (1975)'s analysis of human conversations applies to the communication between experimenters and participants as well as to everyday conversations (e.g. Schwarz, Strack, Hilton, \& Naderer, 1991; Strack, Schwarz, \& Wänke, 1991). Hence, it probably also holds for participants reading stimulus materials in experiments on causal attribution.

In the case of actor-verb-patient messages, the maxim of relation implies that it is noteworthy that this actor did so to this patient (cf. the rarity principle in conditional reasoning, Oaksford \& Chater, 1994; and the greater information contained in rare than in common ones, McKenzie, Ferreira, Mikkelsen, McDermott, \& Skrable, 2001; McKenzie \& Mikkelsen, 2007). If Peter kissed everyone, there would be no need to specify that it was Mary he kissed. If everyone kissed Mary, there would be no need to specify that it was Peter who did so. And if everyone kissed everyone, the message that Peter kissed Mary would be completely meaningless.

In covariation terms, the maxim of relation makes observers spontaneously expect that consensus is low and that distinctiveness is high. Upon encountering explicitly provided contextual information about interpersonal events, they approach the information with these a priori conversation-based expectations in mind (cf. also Hilton, 1995). By consequence, their use of such information towards forming a causal judgment can be described through an attribution question hypothesis, an interdependence hypothesis, and an asymmetry hypothesis.

\section{The attribution question hypothesis}


When people are asked to which extent an actor caused an interpersonal event, Gricean logic dictates that they infer that it is reasonable to assume that the actor contributed to the event. If this were totally implausible, the question would be irrelevant. Similarly, when asked to which extent a patient caused an interpersonal event, Gricean logic dictates that people infer that it is reasonable to assume that the patient contributed to the event. The innuendo effect, implying that media questions make the audience believe that the answers to them must be affirmative, is an illustration of this principle (Wegner, Wenzlaff, Kerker, Beattie, 1981).

When answering questions about actor causation, therefore, participants test the hypothesis that the actor did play a role. When answering questions about patient causation, they test the hypothesis that the patient played a role. Importantly, people test hypotheses in a confirmatory manner (Greenwald, 2012; Hansen, 1980; Nickerson, 1998). Confirming the actor hypothesis requires finding low consensus and low distinctiveness. Already assuming that consensus must be low (as the communicator bothered talking or writing about the event), people focus on distinctiveness. Confirming the patient hypothesis requires finding high consensus and high distinctiveness. Assuming that distinctiveness must be high, people focus on consensus. A Gricean analysis therefore predicts that distinctiveness information affects actor rather than patient attributions and that consensus information affects patient rather than actor attributions.

In fact, there is an additional reason to doubt such a pattern. The contingency judgment literature reveals that people weight in which an object whose frequency or causal role is being judged is present cases more heavily than cases in which it is absent (White, 2002, 2003a,b). When wondering about the role of an actor, observers may scan 
the covariation information for elements mentioning this actor - which is distinctiveness information. When wondering about the role of the patient, they may scan the covariation information for elements mentioning the patient - which is consensus information.

In contrast, the model presents causal attribution as a purely inductive process. People are assumed to collect consensus, distinctiveness, and consistency information without a specific a priori hypothesis guiding their information search and use and hence in a non-directional and rather detached fashion, assess the relevant covariation with the to-be-explained event, and thus decide upon a causal factor (see Hansen, 1980; Lalljee, Lamb, Furnham, \& Jaspars, 1984, for previous criticisms of this aspect of the model).

Research that pits the attribution question hypothesis against the assumption of the covariation model against its other is surprisingly scarce. Researchers have acknowledged and examined the potentially differential role of consensus and distinctiveness. Some results seem to dovetail with predictions from the attribution questions hypothesis, with people above all seeking distinctiveness information while assessing the actor's role and above all seeking consensus information while assessing the patient's causal role (e.g. Alicke \& Insko, 1984; Bassili \& Regan, 1977; Garland, Hardy, \& Stephenson, 1975; Hansen, 1980, Study 3; Hortacsu, 1987, 1990; Major, 1980, Study 1; Pandelaere, Hoorens \& Peeters, 2003). In one study, participants judged their confidence in actor and patient attributions after having received distinctiveness or consensus information. Distinctiveness rather than consensus affected self-reported confidence in actor attributions, with no difference in self-reported confidence in patient attributions (Hansen, 1980, Experiment 4). Still, other results contradict the predications 
from the attribution question hypothesis. For instance, Hilton, Smith, and Alicke (1988, Study 1-4) observed that people above all wished to receive consensus information to answer a attribution question about the actor of an achievement-related event. Yet, all these studies have focused on the extent to which people seek or say that they value various types of covariation information rather than people's actual effect of these types of information on causal judgments. As such they may be relevant about information research preferences but remain silent about actual information use.

The few studies that did test the actual relative use of consensus and distinctiveness information in actor and patient judgments yielded ambiguous results. Besides having participants request as much covariation information as they wished, Major (1980) had participants pass actual causal judgments. In the first experiment (involving an interpersonal event), consensus and distinctiveness affected causal judgments about the actor and the patient. In the second experiment (with a nonsocial event), distinctiveness but not consensus affected both actor and patient judgments. In another study examining the effect of distinctiveness and consensus information on answers to attribution questions about mostly nonsocial events (Hansen, 1980, Study 5), distinctiveness seemed to affect causal judgments about the actor ('person' in Hansen, 1980) more than consensus did and consensus seemed to affect causal judgments about the patient ('entity' in Hansen, 1980) more than distinctiveness did. This is the pattern that the attribution question hypothesis would predict. Unfortunately, neither Major nor Hansen reported statistical tests of the high-low difference being relatively large for distinctiveness effects on actor judgments (as compared to patient judgments) and for consensus effects on patient judgments (as compared to actor judgments).

\section{The interdependence hypothesis}


If a question about actor causation provokes the hypothesis that an actor has caused an event and hence makes observers expect low consensus and low distinctiveness, as a Gricean analysis implies, then high consensus and high distinctiveness contradict their expectations. If a question about patient causation provokes the hypothesis that the patient has caused the event and thus makes observers expect high consensus and high distinctiveness, as the Gricean analysis also implies, then low consensus and low distinctiveness contradict their expectations. Upon encountering unexpected information, observers arguably process the remaining available information more attentively. Thus, consensus and distinctiveness primarily affect actor judgments if distinctiveness and consensus are high, respectively, whereas consensus and distinctiveness primarily affect patient judgments if distinctiveness and consensus are low, respectively.

We thus formulated an interdependence hypothesis stating that both consensus and distinctiveness primarily affect actor judgments if the alternative dimension is high and primarily affect patient judgments if the alternative dimension is low. In the introductory example, observers wishing to assess Peter's causal role in the kissing event may attach more weight to information about how many other people Peter kissed if they learn that many others also kissed Mary than if they learn that only a few people also kissed Mary. Observers wishing to assess Mary’s causal role may weigh information about the number of others who also kissed her more heavily if they learn that Peter kissed many other people than if they learn that Peter kissed few people.

The interdependence hypothesis differs from the idea, implied by the covariation model, that consensus and distinctiveness jointly affect causal judgments but that while they do so consensus affects causal judgments to similar extents over the whole range of 
the distinctiveness dimension and that distinctiveness affects causal judgments to similar extents over the whole range of the consensus dimension. The element of joint determination has previously been described as a consensus and distinctiveness 'interaction' (e.g. Hansen, 1980, Study 5). Yet it merely refers to the idea that the two types of information jointly affect causal judgments. For instance, knowing that Peter kissed few people and that many others kissed Mary jointly suggest that something about Mary makes people want to kiss her. This 'interaction' does not refer to a potentially differential weighting of one type of information under the influence of the other type of information or, stated differently, to observers' use of one dimension being a function of the information they get on the alternative dimension.

To the best of our knowledge, the interdependence hypothesis has not been tested before. Yet, it does have some empirical basis. It has become a truism among perception and social cognition psychologists that context variables affect the perception and evaluation of stimuli. To name one example, observers' interpretation of characteristics attributed to an individual depends on the information they have available about the individual's other characteristics (for an overview, see Aronson, 2012). We therefore found it worthwhile to examine whether a similar interdependence also characterizes people's use of consensus and distinctiveness information in causal judgments.

\section{The Asymmetry hypothesis}

If verbal descriptions of interpersonal events make people assume low consensus and high distinctiveness, as a Gricean analysis implies, then explicitly provided covariation information about low consensus or high distinctiveness contributes little new information. Hence, it will not strongly affect causal judgments. In contrast, information about consensus being high or distinctiveness being low goes beyond (and contradicts) 
what the observers already know. Hence, it will affect their judgments. We thus formulated an asymmetry hypothesis stating that high consensus and low distinctiveness affect causal judgments more than low consensus and high distinctiveness do.

One might object that people also apply the maxim of quantity on the covariation information, and hence always assumes that is informative. Yet participants do not view the covariation information in the same manner as the behavioral descriptions themselves. They view the descriptions as the to-be-explained events and the covariation information as additional information that may or may not be useful towards the explanation task. They thus view the latter as information whose relevance they need to assess as part of the experimental task (cf. Hilton \& Slugoski, 1986).

The asymmetry hypothesis is obviously at odds with the idea implied by the presentation of the covariation model as a perfect cube, namely, that each position on each dimension is equally informative about the causation going on. For instance, knowing that many people kiss Mary is, according to the model, as informative as knowing that few people kiss Mary - but it in different directions. In contrast, the asymmetry hypothesis states that the former message, including more unexpected information, tells more than the latter.

One study may, at first sight, seem at odds with the asymmetry hypothesis. Hilton and Slugoski (1986, Study 1) had participants judge how much information they felt various constellations of covariation conveyed about the actor, the patient (in their terminology: the stimulus), and the circumstances. When the behaviors were not strongly norm-dictated or norm-deviating, participants reported that low consensus was more informative about the actor than high consensus and that high distinctiveness was more informative about the patient than low distinctiveness. This pattern is exactly the 
opposite of what the asymmetry hypothesis states. Yet, Hilton and Slugovki examined evaluative judgments about the covariation information rather than the effect of this information on causal judgments. Assuming that processes guiding the judgment of data are not necessarily identical to those guiding actual causal judgments, it becomes clear that the assumption of symmetry in actual causal judgments has basically gone untested. Published studies typically included high versus low consensus and high versus low distinctiveness without control conditions without covariation information. In a rare exception Hansen (1980, Study 5) compared causal judgments under low and high consensus and distinctiveness as compared to the situation in which the relevant covariation information was absent. As the covariation model predicts, he found opposing effects of low and high consensus and distinctiveness. Yet, he did not test whether they affected causal judgments to similar extents.

\section{The Present Research}

The covariation model is widely recognized as a landmark theory in causal attribution (cf. Weiner, 2008). Many theories of causal judgments extended, articulated, complemented, or built upon it (e.g, Bowler, Woehr, Bowler, Wuensch, \& McIntyre, 2011; Cheng, 1997; Eberly, Halley, Johnson, \& Mitchell, 2011; Green \& Mitchell, 1979; Hilton, Smith, \& Kim, 1995; Martinko \& Thomson, 1997; Sutton \& McClure, 2001; Van Overwalle, 1997, 2003; White, 2002, 2003a,b). There is even a recently increased interest in the model, both in applied psychological disciplines (e.g., Harris \& Fiske, 2008; Klein, Apple, \& Kahn, 2011; Quayle \& Naidoo, 2012; Samnami, Singh, \& Ezzedeen, 2013; Schwarz, 2012; Schimansky, Rössler, \& Haker 2012; Seiver, Gopnik, \& Goodman, 2013) and in fields outside of psychology (e.g., Coombs, 2007; Jiang, Gretzel, \& Law, 2010; Koonce, Seybert, \& Smith, 2011; McClure, Allen, \& Wolkey, 
2001; Ployhart, Ehrhart, \& Hayes, 2005; Rees, Ingledew, \& Hardy, 2005; Ryan \& Boscia, 2003; Schwarz, 2008). All these recent developments make it timely to examine its underlying assumptions. Inspired by a Gricean analysis, we tested an attribution question hypothesis, an interdependence hypothesis, and an asymmetry hypothesis for causal judgments about actors and patients of verbally described interpersonal events.

Besides its Gricean approach, our research is characterized by its focus on interpersonal events. Failing to distinguish between social and nonsocial events, most previous tests of aspects of the covariation model have mostly used nonsocial ones. Yet, people are likely to approach attribution tasks depending on the social nature of the tobe-explained events. First, patients of interpersonal events have a will of their own and hence may be suspected of intentionally provoking the actors' behaviors. Second, people are mostly interested in other individuals' interpersonal behaviors. Knowing how another individual treats fellow human beings and how others treat the individual informs the observer about how he or she is likely to be treated. Supporting this reasoning, person judgments on the dimension of warmth (rather than competence) dominate person perception (e.g. Fiske, Cuddy, \& Glick, 2007; Wojciszke \& Abele, 2008).

Only two projects have compared causal attributions for nonsocial and interpersonal events. McArthur (1976) examined attributions for events of which the actor and the patient were persons or inanimate objects. She found no effects of the patient being a person or an object. Yet, an inspection of her stimuli reveals that the meaning of the verbs covaried with the person-object variables. Orvis, Cunningham, and Kelley (1975) examined how people filled in incomplete covariation information or chose between 
causal candidates. Their participants more often inferred low distinctiveness, high consensus, and high consistency for achievements than for interpersonal events.

Two additional sets of studies yielded relevant results. Major (1980) presented participants with a vignette about an interpersonal event (a fight, Experiment 1) or a nonsocial event (an employee working on a project, Experiment 2). Before passing causal judgments, participants could request covariation information. Participants in the first experiment requested more distinctiveness than consensus information whereas participants in the second experiment requested equal amounts of both. Hansen (1980, Experiment 1) had participants generate causal hypotheses about achievements, emotions, and therapeutic outcomes. The latter category, the sole that exclusively consisted of interpersonal events, provoked hypotheses about actors and patients (clients and therapists) whereas the former two categories mainly provoked hypotheses about either actors or 'patients', respectively. Moreover, therapeutic outcomes were more often attributed to both the actor and the patient. For all these reasons, we submit that it is worth the effort to specifically study the assumptions of the covariation model as it applies to interpersonal events.

Now that the general context of our research is set, it is time to present the blueprint of our studies. All three studies tested the attribution question hypothesis and the interdependence hypothesis. Study 2 and Study 3 also tested the asymmetry hypothesis. Participants always read actor-verb-patient descriptions of interpersonal events. In Study 1, the actor-verb-patient descriptions went accompanied by high versus low consensus and distinctiveness information. Study 2 and Study 3 included control conditions without covariation information. In Study 3 we also manipulated consistency information (low, high, absent). As we were interested in how consensus and 
distinctiveness affected actual causal judgments, rather than in judgments about how informative people considered consensus and distinctiveness and how interested they were in obtaining the information, we had participant indicated how much the actor had contributed to the event and how much the patient had contributed to the event. These questions were modeled after those used by Hansen (1980, Experiment 2).

We used rating scales instead of forced-choice questions for three different reasons. First and foremost, testing our hypotheses necessitated that we examined actor and patient attributions separately. Second, forced-choice measures rest upon the assumption that attributions are inversely related, with increasing attributions to one causal agent necessitating the role of another agent being discounted - an assumption that has not received consistent empirical support (Hansen, 1980; Miller, Smith, \& Uleman, 1981; Solomon, 1978). Forced- choice studies typically allow participants to choose between an attribution to the actor, the patient, the situation, and the actorpatient interaction (sometimes also including various other interactions). We had participants judge the causal role of the actor and the patient only. Besides being tedious, having to answer four or more questions per stimulus description would probably have made participants weary about us being interested in the internal logic of their answers. Moreover, our approach did not prohibit situational or interactional causation to become evident. To show that they viewed the actor-patient combination as the main causal agent, for instance, participants could give high ratings for both the actor and the patient. To show that neither the actor nor the patient was the main causal agent, they could give low ratings for both.

\section{Study 1}


The attribution question hypothesis predicted that participants would predominantly use distinctiveness information to pass causal judgments on the actor and use consensus information to pass causal judgments on the patient. The interdependence hypothesis predicted that participants' use of consensus and distinctiveness should depend on the information about the alternative dimension.

\section{Method}

Participants. Eighty first-year psychology students (65 women, $M_{\text {age }}=18.3$ years, age range: 17-26 years) participated to partially fulfill course requirements.

Materials. Each participant received a questionnaire with all instructions and stimulus materials. The instructions read that the study was about how people interpret interpersonal events when they possess limited information. Participants would therefore read actor-verb-patient descriptions along with some context information, and answer questions about them. The instructions acknowledged that the task might be difficult yet encouraged participants to not skip any questions.

Participants then saw the actor-verb-patient descriptions. Each description went accompanied by information indicating high versus low consensus and distinctiveness. We thus created four covariation combinations. For instance, one item might read:

\section{Ann hits Bart.}

Ann hits almost everyone. Almost everyone hits Bart.

The actors and the patients were indicated by common first names, with different names for each description. To ensure generality, half of the events were desirable and the other half were undesirable. Moreover, the descriptions included equal numbers of descriptive action verbs (e.g. embrace, kick), interpretative action verbs (e.g. help, provoke), and state verbs (e.g. trust, fear) to control for implicit consensus and 
distinctiveness information participants might derive from different verb types (cf. Hoorens, Maier, \& Maris, 2012; Rudolph \& Försterling, 1997; Rudolph \& von Hecker, 2006; Semin \& Fiedler, 1988). Two questions appeared under each stimulus sentence: 'To what extent was the event caused by the actor?', and 'To what extent was the event caused by the patient?' Participants answered using 9-point scales $(1=$ not at all and $9=$ entirely).

Procedure and design. We ran the experiment during two mass testing sessions. Each participant received a questionnaire that they filled out in their own pace.

The combination of a high versus low level on two dimensions of covariation information with two attribution questions resulted in a 2 (Consensus: low vs. high) x 2 (Distinctiveness: low vs. high) x 2 (Target: actor vs. patient) within-subjects design. Each condition was represented by 18 verbs ( 3 positive and 3 negative instances of 3 verb types) taken from Hoorens et al. (2012). Each participant thus responded to 72 items (18 descriptions x 4 consensus-distinctiveness combinations).

The verbs appeared in a fixed random order with the restriction that each verb occurred once before any given verb occurred again. We counterbalanced the order of consensus and distinctiveness, the order of the questions, and the description's gender composition (with half of the participants reading about female actors and male patients and the other half reading about male actors and female patients).

\section{Results and discussion}

If participants inadvertently treated the questions as complementary, assuming that actor and patient ratings should be inversely related, then the two scales would boil down to a single bipolar scale and the two items would be very strongly negatively 
correlated. Instead, we observed a modest positive correlation between mean actor ratings and mean patient ratings $(r=0.29, d f=78 ; p=.009)$.

Thus reassured we calculated average causal judgments per Consensus $\mathrm{x}$ Distinctiveness $x$ Target combination. We subjected these to an ANOVA with consensus, distinctiveness, and target as within-subject variables. The main effects of consensus, $F(1,79)=60.0, p<.001, \eta^{2}=.432$, distinctiveness, $F(1,79)=9.4, p=.003$, $\eta^{2}=.107$, and target, $F(1,79)=48.9, p<.001, \eta^{2}=.382$, were significant. All three factors were involved in interactions that will be discussed below. The cell means of this and the following studies are presented in Table 1.

Table 1 about here

The attribution question hypothesis. The attribution question hypothesis states that actor judgments predominantly depend on distinctiveness whereas patient judgments predominantly depend on consensus. The predicted two-way interactions were significant: target by consensus, $F(1,79)=127.2, p<.001, \eta^{2}=.617$, and target by distinctiveness, $F(1,79)=133.8, p<.001, \eta^{2}=.629$. To follow up on them we calculated low-high consensus and low-high distinctiveness difference scores. As expected, consensus differences were larger for patient judgments $(M=1.6, S D=1.3)$ than for actor judgments $(M=0.6, S D=0.8), t(79)=7.7, p<.001$, and distinctiveness differences were larger for actor judgments $(M=1.4, S D=1.4)$ than for patient judgments $(M=0.9, S D=0.8), t(79)=3.1, p=.003$. Another way to look at the interaction is by saying that for actor judgments distinctiveness differences exceeded $(M$ $=1.4, S D=1.4)$ consensus differences $(M=0.6, S D=0.8), t(79)=6.4, p<.001$, 
whereas for patient judgments consensus differences exceeded $(M=1.6, S D=1.3)$ distinctiveness differences $(M=0.9, S D=0.8), t(79)=5.2, p<.001$.

Our findings thus supported the attribution question hypothesis. Extending the findings of Hansen (1980, Study 5), they demonstrated the predicted unequal use of consensus and distinctiveness information to answer attribution questions about the actor and the patient of interpersonal events.

The interdependence hypothesis. The interdependence hypothesis predicted that consensus and distinctiveness would particularly affect causal judgments about the actor if the alternative dimension was high and particularly affect causal judgments about the patient if the alternative dimension was low.

The predicted two-way interaction of consensus and distinctiveness and three-way interaction of target, consensus, and distinctiveness were significant, $F(1,79)=35.8, p<$ $.001, \eta^{2}=.312$, and $F(1,79)=11.1, p=.001, \eta^{2}=.123$, respectively. To understand these interactions we calculated low-high difference scores reflecting how much a given dimension affected causal judgments (see Figure 1). For actor judgments, the low-high differences were larger if the alternative dimension was high than if the alternative dimension was low; $t$ 's(79) $=-3.9, p<.001$. For patient judgments, the low-high differences were larger if the alternative dimension was low than if the alternative dimension was high; $t^{\prime} s(79)=6.1, p<.001$. Study 1 thus supported the interdependence hypothesis.

Figure 1 about here

\section{Study 2}


Study 2 aimed at replicating the findings of Study 1 while testing the asymmetry hypothesis.

\section{Method}

Participants. First year students participated to fulfill a course requirement (108 women $M_{\text {age }}=18$ years, age range: $17-21$ years).

Materials, procedure, and design. Study 2 was identical to Study 1 except for the following. First, consensus and distinctiveness were either high, low, or absent. Second, we compensated the increased number of combinations by counterbalancing verb category over participants. Each participant thus responded to 54 items (6 descriptions with 9 consensus-distinctiveness combinations) representing 3 (Consensus: low vs. high vs. none) x 3 (Distinctiveness: low vs. high vs. none) x 2 (Target: actor vs. patient) within-subjects conditions.

\section{Results and discussion}

There was a significant but low negative correlation between the actor and the patient ratings $(r=-0.19, d f=111 ; p=.044)$. We therefore treated the causal judgments as in Study 1. The ANOVA with target, consensus, and distinctiveness as within-subject variables yielded main effects of target, $F(1,112)=67.29, p<.001, \eta^{2}=.38$, and consensus, $F(2,224)=30.22, p<.001, \eta^{2}=.21$. Both factors were involved in the interactions discussed below.

The attribution question hypothesis. Participants used consensus information more to assess the causal role of the patient than to assess the causal role of the actor. The interaction of target by consensus was significant, $F(2,224)=281.53, p<.001, \eta^{2}=$ .72. The high-low consensus difference was larger for patient judgments $\left(\left|M_{\text {diff }}\right|=2.42\right.$, $S D=1.67)$ than for actor judgments $\left(\left|M_{\text {diff }}\right|=1.55, S D=1.04\right), t(112)=6.1, p<.001$. 
Yet, participants did not use distinctiveness information more to assess the causal role of the actor than to assess the causal role of the patient. The interaction of target by distinctiveness was significant, $F(2,224)=229.54, p<.001, \eta^{2}=.67$, yet the high-low distinctiveness difference was not larger for actor judgments $\left(\left|M_{\text {diff }}\right|=1.59, S D=1.58\right)$ than for patient judgments $\left(\left|M_{\text {diff }}\right|=1.80, S D=0.97\right), \mathrm{t}(112)=1.5, \mathrm{p}=.147$.

Study 2 thus yielded partial support for the attribution question hypothesis. As in Study 1 , the attribution question affected the use of consensus information. Contrary to Study 1 , we found no evidence of the attribution question affecting the use of distinctiveness information.

The interdependence hypothesis. As in Study 1, a two-way interaction of consensus by distinctiveness, $F(4,448)=52.73, p<.001, \eta^{2}=.32$, was qualified by a predicted three-way with target, $F(4,448)=88.53, p<.001, \eta^{2}=.44$. For actor judgments, the low-high differences were larger if the alternative dimension was high than if the alternative dimension was low; $t$ 's $(112)=4.9, \mathrm{p}<.001$. For patient judgments, the low-high differences were larger of the alternative dimension was low than if the alternative dimension was high; $t^{\prime} \mathrm{s}(112)=5.9, \mathrm{p}<.001$. Consensus affected judgments most in the absence of distinctiveness (actor: $\left|M_{\text {diff }}\right|=2.59, S D=1.63$; patient: $\left.\left|M_{\text {diff }}\right|=2.89, S D=1.85\right)$, and distinctiveness affected judgments most in the absence of consensus (actor: $\left|M_{d i f f}\right|=2.08, S D=1.82$; patient: $\left|M_{\text {diff }}\right|=3.35, S D=1.85$ ).

The interdependence hypothesis was thus fully supported. Consensus and distinctiveness primarily affected causal judgments about the actor if the alternative dimension was high and primarily affected causal judgments about the patient if the alternative dimension was low. Both consensus and distinctiveness affected causal judgments most of all in the absence of information about the alternative dimension. 
The asymmetry hypothesis. We tested the prediction that high consensus and low distinctiveness would affect causal judgments more than low consensus and high distinctiveness would. As compared to the no-consensus-condition low consensus enhanced actor judgments $\left(M_{\text {diff }}=0.58, S D=.75\right), t(112)=8.25, p<.001$, and reduced patient judgments $\left(M_{\text {diff }}=-0.76, S D=1.05\right), t(112)=7.68, p<.001$, whereas high consensus reduced actor judgments $\left(M_{\text {diff }}=-0.97, S D=.80\right), t(112)=12.87, p<.001$, and enhanced patient judgments $\left(M_{\text {diff }}=1.66, S D=1.05\right), t(112)=16.85, p<.001$. Similarly, as compared to the no-distinctiveness-condition low distinctiveness enhanced actor judgments $\left(M_{\text {diff }}=0.96, S D=1.11\right), t(112)=9.17, p<.001$, and reduced patient judgments $\left(M_{\text {diff }}=-1.03, S D=0.74\right), t(112)=14.91, p<.001$, whereas high distinctiveness reduced actor judgments $\left(M_{\text {diff }}=-0.63, S D=0.86\right), t(112)=7.77, p<$ .001 , and enhanced patient judgments $\left(M_{\text {diff }}=0.76, S D=0.66\right), t(112)=12.39, p<.001$. Consistent with the asymmetry hypothesis, a comparison of the low-no consensus and high-no consensus differences revealed that it was particularly high consensus that affected actor judgments, $t(112)=3.6, p=.001$, and patient judgments, $\mathrm{t}(112)=7.6, p<$ .001. A comparison of the low-no distinctiveness and high-no distinctiveness differences revealed that it was particularly low distinctiveness that affected actor judgments, $t(112)=2.9, p=.005$, and patient judgments, $\mathrm{t}(112)=2.8, p=.005$. Study 2 thus supported the asymmetry hypothesis.

\section{Study 3}

Study 3 tested the attribution question hypothesis, the interdependence hypothesis, and the asymmetry hypothesis in a design that also included a consistency manipulation. It thus allowed testing the generality of the findings in Study 1 and Study 2.

\section{Method}


Participants. First year students participated to fulfill a course requirement (55 women $M_{\text {age }}=19$ years, age range: $17-22$ years).

Materials, procedure, and design. Study 3 was identical to Study 2 except for the following. First, consistency was high, low, or absent. Second, as verb category did not markedly influence causal attributions in the previous studies each participant received descriptions with interpretative action verbs. Third, to compensate the increased number of covariation patterns we counterbalanced valence over actors. Each participant thus responded to 81 items (3 descriptions with 27 consistency-consensus-distinctiveness combinations each) representing 3 (Consistency: low vs. high vs. none) x 3 (Consensus: low vs. high vs. none) x 3 (Distinctiveness: low vs. high vs. none) x 2 (Target: actor vs. patient) within-subjects conditions.

\section{Results and Discussion}

The actor and the patient ratings were significantly negatively correlated $(r=-0.53, d f=$ $68 ; p=.001)$. We postpone a discussion of this correlation until the General Discussion. As it still fell short of the correlation one would expect if the subject and object ratings tapped into the same construct we decided to treated the results as in the previous studies.

Study 3 mainly examined whether the effects that we observed in Study 1 and Study 2 also emerged if we varied consistency information. Consistency does not differentiate between actor and patient attributions, so we were interested in replicating the effects of Study 1 and Study 2 rather than in analyzing consistency effects. We therefore start by describing the results as they pertain to our hypotheses and describe consistency effects towards the end. 
The ANOVA with target, consistency, consensus, and distinctiveness as withinsubject variables yielded main effects of target, $F(1,69)=83.23, p<.001, \eta^{2}=.55$, consistency, $F(2,138)=12.19, p<.001, \eta^{2}=.15$, consensus, $F(2,138)=11.9, p<.001$, $\eta^{2}=.15$, and distinctiveness, $F(2,138)=3.16, p=.05, \eta^{2}=.04$. All these factors were involved in higher order interactions explained below.

The attribution question hypothesis. Participants used consensus information more to assess the causal role of the patient than to assess the causal role of the actor. The interaction of target by consensus was significant, $F(2,138)=46.3, p<.001, \eta^{2}=$ .40 , with the high-low consensus difference being larger for patient judgments $\left(\left|M_{d i f f}\right|=\right.$ $1.15, S D=1.6)$ than for actor judgments $\left(\left|M_{\text {diff }}\right|=.60, S D=.8\right), t(69)=3.5, p=.001$. In contrast, participants did not use distinctiveness information more to assess the causal role of the actor than to assess the causal role of the patient. The interaction of target by distinctiveness was significant, $F(2,138)=38.4, p<.001, \eta^{2}=.36$, yet the high-low distinctiveness difference was not larger for actor judgments $\left(\left|M_{\text {diff }}\right|=.62, S D=1.3\right)$ than for patient judgments $\left(\left|M_{d i f f}\right|=.87, S D=0.8\right), \mathrm{t}(69)=1.8, \mathrm{p}=.081$. Study 3 thus yielded partial support for the attribution question hypothesis.

The interdependence hypothesis. The two-way interaction of consensus by distinctiveness, $F(4,276)=8.5, p<.001, \eta^{2}=.11$, was again qualified by a three-way with target, $F(4,276)=14.6, p<.001, \eta^{2}=.18$.

Consensus affected actor judgments more if distinctiveness information was absent $\left(\left|M_{\text {diff }}\right|=.95, S D=1.2\right)$ than if distinctiveness was low $\left(\left|M_{\text {diff }}\right|=0.41, S D=1.0\right)$ or high $\left(\left|M_{\text {diff }}\right|=0.44, S D=.9\right), t(69)=3.6, p=.001$, and $t(69)=3.8, p<.001$, respectively. The latter conditions did not differ, $t(69)=-.2, p=.848$. Neither did the effect of distinctiveness on actor judgments depend on consensus (high: $\left|M_{\text {diff }}\right|=.57, S D=1.5$; 
low: $\left|M_{\text {diff }}\right|=.75, S D=1.5$; absent: $\left.\left|M_{\text {diff }}\right|=.55, S D=1.4\right)$, all $t$ 's $(69)<1.6$, all $p$ 's $>.115$. Consensus affected patient judgments more if distinctiveness information was absent $\left(\left|M_{\text {diff }}\right|=1.48, S D=1.6\right)$ or if distinctiveness was low $\left(\left|M_{\text {diff }}\right|=1.31, S D=1.9\right)$ than if it was high $\left(\left|M_{d i f f}\right|=.66, S D=1.7\right), t(69)=5.2, p<.001$, and, $t(69)=4.6, p<.001$, respectively. The former conditions did not differ, $t(69)=1.2, p=.223$. Distinctiveness affected patient judgments more if consensus information was absent $\left(\left|M_{\text {diff }}\right|=1.35, S D\right.$ $=1.5)$ than if it was low $\left(\left|M_{\text {diff }}\right|=.95, S D=1.0\right), t(69)=2.9, p=.004$, and more if consensus was low than if it was high $\left(\left|M_{d i f f}\right|=.31, S D=.78\right), t(69)=4.6, p<.001$.

Study 3 thus yielded partial support for the interdependence hypothesis. Consensus and distinctiveness affected patient judgments more if the alternative dimension was low than if it was high. Yet, they did not affect actor judgments more if the alternative dimension was high than if it was low.

The asymmetry hypothesis. Low consensus enhanced actor judgments $\left(M_{\text {diff }}=\right.$ $0.13, S D=.4), t(69)=2.71, p=.009$, and reduced patient judgments $\left(M_{\text {diff }}=-0.20, S D=\right.$ $.7), t(69)=2.29, p=.025$, whereas high consensus reduced actor judgments $\left(M_{\text {diff }}=-\right.$ $0.47, S D=.7), t(69)=6.01, p<.001$, and enhanced patient judgments $\left(M_{\text {diff }}=.96, S D=\right.$ $1.2), t(69)=6.90, p<.001$. Low distinctiveness enhanced actor judgments $\left(M_{\text {diff }}=0.48\right.$, $S D=.9), t(69)=4.67, p<.001$, and reduced patient judgments $\left(M_{\text {diff }}=-.53, S D=0.6\right)$, $t(69)=7.63, p<.001$, whereas high distinctiveness marginally reduced actor judgments $\left(M_{\text {diff }}=-0.14, S D=0.7\right), t(69)=1.76, p=.083$, and enhanced patient judgments $\left(M_{\text {diff }}=\right.$ $0.34, S D=0.5), t(69)=5.56, p<.001$. Consistent with the asymmetry hypothesis, however, participants used high consensus information more than low consensus information, both in actor judgments, $t(69)=3.7, p<.001$, and in patient judgments, $\mathrm{t}(69)=5.8, p<.001$. Similarly, they used low distinctiveness information more than 
they used high distinctiveness information, both in actor judgments, $t(69)=3.7, p<$ .001 , and in patient judgments, $\mathrm{t}(69)=2.2, p=.031$. Study 3 thus uniformly supported the asymmetry hypothesis.

The influence of consistency. As we were not primarily interested in effects involving consistency, we merely include them for completeness' sake.

There was an interaction of target by consistency, $F(2,138)=8.3, p<.001, \eta^{2}=.11$. High consistency enhanced actor judgments $\left(M_{d i f}=.13, S D=.4\right), t(69)=2.86, p=.006$, and low consistency reduced them $\left(M_{\text {dif }}=-.27, S D=.6\right), t(69)=3.88, p<.001$. Consistency did not influence patient judgments (high: $M_{d i f}=.06, S D=.4, t(69)=1.25$, $p=.216 ;$ low: $\left.M_{d i f}=.10, S D=.5, t(69)=1.57, p=.120\right)$.

The interaction of consistency by consensus, distinctiveness, and target was significant, $F(8,552)=4.9, p<.001, \eta^{2}=.07$. Interactions of consistency by consensus, $F(4,276)=3.5, p=.008, \eta^{2}=.05$, and of consistency by distinctiveness, $F(4,276)=3.4$, $p<.001, \eta^{2}=.07$, were each qualified by a higher order interaction with target, $F(4,276)=11.1, p<.001, \eta^{2}=.14$ and $F(4,276)=3.7, p=.006, \eta^{2}=.05$, respectively.

Consistency influenced the use of low but not of high consensus. Participants used low consensus for actor judgments if consistency was absent $\left(M_{\text {diff }}=.21, S D=.53\right)$, $t(69)=3.24, p=.002$, but not if it was present (high consistency: $M_{d i f f}=.12, S D=.59$, $t(69)=1.77, p=.082 ;$ low consistency: $\left.M_{\text {diff }}=.06, S D=.73, t(69)=.64, p=.523\right)$. They used low consensus for patient judgments if consistency was low $\left(M_{\text {diff }}=-.32, S D\right.$ $=.95), t(69)=2.81, p=.006$, but not if it was high $\left(M_{\text {diff }}=-.23, S D=.79\right), t(69)=2.43$, $p=.018$, or absent $\left(M_{\text {diff }}=-.04, S D=.91\right), t(69)=.39, p=.696$. The use of high consensus did not depend on consistency for actor judgments (low: $M_{\text {diff }}=-.25, S D=$ .87 ; high: $M_{\text {diff }}=-.50, S D=.58$; absent: $\left.M_{\text {diff }}=-.67, S D=.97\right)$, all $t$ 's $(69)>2.39, p$ 's < 
.020 , or patient judgments (low: $M_{\text {diff }}=.61, S D=1.33$; high: $M_{\text {diff }}=1.03, S D=1.21$; absent: $\left.M_{\text {diff }}=1.22, S D=1.27\right)$, all $t$ 's $(69)>3.85, p$ 's $<.001$.

Consistency influenced the use of high but not of low distinctiveness. Participants failed to use high distinctiveness for actor judgments if consistency was low or absent (low: $M_{\text {diff }}=-.05, S D=.70$; absent: $M_{\text {diff }}=-.17, S D=1.00$ ), all $t$ 's(69) $\langle 1.38, p$ 's $>$ .170 , but did use it if consistency was high $\left(M_{\text {diff }}=-.21, S D=.86\right), t(69)=2.02, p=$ .047. Participants also used high distinctiveness for patient judgments if consistency was high or absent (high: $M_{\text {diff }}=.32, S D=.89$; absent: $M_{\text {diff }}=.55, S D=.78$ ), all $t$ 's(69) $<3.02, p$ 's $<.004$, but not if it was low $\left(M_{\text {diff }}=.16, S D=.75\right), t(69)<1.83, p=.072$. Consistency did not affect the use of low distinctiveness, either for actor judgments (low: $M_{\text {diff }}=.54, S D=1.12$; high: $M_{\text {diff }}=.36, S D=.84$; absent: $M_{\text {diff }}=.53, S D=1.08$ ), all $t$ 's(69) > 3.63, $p$ 's $<.001$, or for patient judgments (low: $M_{\text {diff }}=-.40, S D=.87$; high: $M_{\text {diff }}=-.55, S D=.804 ;$ absent: $\left.M_{\text {diff }}=-.64, S D=.84\right)$, all $t$ 's $(69)>3.80, p$ 's $<.001$.

\section{General Discussion}

Based on a Gricean analysis, we challenged three basic assumptions of the covariation model as it applies to causal attributions for verbally described interpersonal events. We found that the assumptions of invariant, independent, and symmetrical use of consensus and distinctiveness information did not generally hold, at least not in this specific attribution case.

The attribution question hypothesis, saying that people particularly use distinctiveness information to judge the causal role of the actor and consensus information to judge the causal role of the patient, received partial support. Participants consistently weighted consensus heavier while judging the patient's role than while judging the actor's role (Study 1, $2 \& 3$ ). In one study (Study 1), but not in the two other 
studies, they also weighted distinctiveness heavier while judging the actor's role than while judging the patient's role.

The interdependence hypothesis, saying that the use of a given covariation dimension critically depends on the alternative dimension, was generally supported. In two studies (Study $1 \& 2$ ), but not in the third one, consensus and distinctiveness particularly affected actor judgments if the alternative dimension was high. In all three studies, consensus and distinctiveness particularly affected patient judgments if the alternative dimension was low.

Moreover, both dimensions affected judgments most when information about the alternative dimension was absent (Study $2 \& 3$ ). The latter finding might seem trivial: If no other information is available, it seems logical that observers use the information they do have more heavily. Yet our finding is relevant in the light of Rose et al. (2011) who found that people use consensus information more when they are aware of a potential situational explanation for an event than when they are not aware of such an explanation. From the actor's point of view, patient causation is a type of situational causation. Rose et al.'s finding might therefore be interpreted as showing that consensus information affects causal attributions more in the presence of distinctiveness information than in its absence. Showing the opposite, our findings support Rose et al. (2011)'s interpretation in terms of an effect of information about a causal mechanism and not of an effect of general situational information.

The asymmetry hypothesis was consistently supported. Two of our studies put it to test (Study 2 \& 3). As Grice's logic of conversations implies, in both studies high consensus and low distinctiveness influenced causal judgments more than low consensus and high distinctiveness did. 
Although our research aimed at testing three specific hypotheses about the use of different types of covariation information, it broadly adds to the general literature on the relative use and search of consensus, distinctiveness, and consistency. Most research on the relative search for consensus versus distinctiveness information originated in early studies suggesting that distinctiveness affected causal judgments more than consensus did (Bruce, Cunningham, \& Kelley, 1975; DiVitto \& McArthur, 1978; Kassin, 1979; McArthur, 1972, 1976; Wright, Lüüs \& Christie, 1990; see, however, Försterling, 1989; Iacobucci \& McGill, 1990). Later studies revealed that the underuse of consensus was by far not as general as once thought (e.g. Hansen \& Donoghue, 1977; Hilton, Smith, \& Alicke, 1988; Quayle \& Naidoo, 2012; Rose, Windschitl, \& Jenson, 2011; Ruble \& Feldman, 1976; Wells \& Harvey, 1977). It was suggested to depend on, among other factors, on the nature of the attribution question being answered. We showed that predictable regularities exist not only in the search and evaluation of covariation information but also in its actual use.

\section{Implications and Suggestions for Further Research}

We did not strive to test the general validity of Kelley's covariation principle or to challenge alternative formulations (e.g. Cheng, 1997; Cheng \& Novick, 1990, 1992; Hewstone \& Jaspars, 1987; Hilton, 2007; Hilton \& Jaspars, 1987; Hilton \& Sluhosky, 1986; Jaspars, 1983; Jaspars, Hewstone, \& Fincham, 1983; see also Perales \& Shanks, 2003). We merely tested, from a Gricean point of view, whether the implicit assumptions of the covariation model about the use of covariation information characterize actual causal judgments of verbally described interpersonal events. One conclusion from our findings is that the standard presentation of the model as a perfect cube cannot generally be maintained for these particular stimulus events. The cube 
presentation suggests that the assumptions of equal, independent, and symmetrical use are met by default. We showed that, for interpersonal events communicated through verbal descriptions, this is not sufficiently true to warrant such a generalization. Our research thus shows that applications of the covariation model may profit from a Gricean complement. Adding such a complement, at least in the case of attributions for verbally communicated events, places the attribution process within the conversational context that often surrounds it. Moreover, it allows explaining attribution patterns that are otherwise hard to understand.

On a more speculative level, our research implies a plea for a clear distinction between various aspects of the attribution process. Our reading of the literature suggested that causal judgments, information searches, and information judgments are often taken as different measurements of the same underlying process. Yet, judgments of the normative influence of covariation information may not tap into the same behavior as actual causal judgments do. It would therefore be interesting to examine whether the covariation model mainly holds as a model of actual causal attributions versus as a model for (normative or descriptive) lay beliefs about causal attribution.

Also on a speculative level, our findings plea for the desirability of a clear distinction, in future research, between social and nonsocial events to be used as stimuli in causal attribution studies. Previous tests supporting the covariation model have mostly used nonsocial events. Our research, uniquely including social events, cast doubt on some of the model's basic assumptions being always followed. It thus points at the possibility that people explain social versus nonsocial events differently. Taken to its extreme, the covariation model, though mostly used to understand how people interpret social interactions, may above all hold for nonsocial events. 
Our suggestions for future research follow from these implications. We recommend that studies on causal attribution systematically compare attribution processes for social and nonsocial events and carefully distinguish between the various aspects of attribution processes. Most of all, however, we recommend further explorations of how conversational contexts may affect attribution processes.

\section{Limitations}

One obvious limitation of our research was that the results were not totally consistent. The support for the attribution question hypothesis was less uniform in Studies 2-3 than in Study 1. The support for the interdependence hypothesis was less uniform in Study 3 than in Studies 1-2.

One explanation for the poorer support for the interdependence hypothesis in Study 3 than in Studies 1-2 may be that, upon encountering unexpected consensus or distinctiveness information, participants in Study 3 could devote part of their enhanced attention to the consistency information. Despite the observed evidence for the attribution question and the interdependence hypothesis being not as uniform as the evidence for the asymmetry hypothesis, however, the overall pattern of results was strong enough to doubt whether the assumptions underlying the covariation model hold in the case of causal judgments about verbally described interpersonal events.

One more general explanation that could account for all inconsistencies may be based on the increasing repetitiveness of the stimuli over studies. Participants in Study 1 saw 18 events with four covariation combinations. Those in Study 2 responded to six events with nine combinations. Participants in Study 3 responded to three events in 27 combinations. The increasing repetitiveness may have created increasing levels of boredom or confusion and thus have rendered it difficult for significant effects to occur. 
This issue may in future studies be addressed by using larger samples of participants, each causally judging a limited set of events.

It is probably also the additional manipulation of levels (from Study 1 to Study 2) and consistency information (from Study 2 to Study 3) that made the subject and object judgments correlate ever more negatively. It will be remembered that Experiment 3 was the sole study to yield a considerable correlation between object and subject ratings (be it not a perfect one, and even not a correlation that would psychometrically warrant the combination of items). So why did participants in Study 3 treat the subject and object to a larger extent as complementary causal agents than participants in Study 1 and Study 2 did? One explanation may be that Study 3 was the sole study in which there were no fewer than three covariation dimensions (consensus, distinctiveness, consistency) of which participants encountered three levels of information (high, low, absent), each combination of covariation information to be applied to each stimulus event. This encompassing set-up may have provoked the idea among participants that they encountered all possible combinations that a researcher could reasonably come up with and that such rendered it possible to evaluate whether their responses were internally consistent. They may therefore have tried to be as consistent and logical as possible in their answers, thus giving subject and object ratings that were strongly negatively correlated.

A completely different limitation of our research is that we examined causal judgments rather than asking open questions about the causes of interpersonal events. This choice was inspired by one of the hypotheses being tested (namely, the attribution question hypothesis). At the same time, it was consistent with previous research on the covariation model in particular and causal attribution in general. Future research may 
examine to which extent asymmetry and interdependence phenomena also occur in idiosyncratic causal attributions.

In addition, we examined how people use different types of covariation information when they do use covariation information. Whereas people may and sometimes do use covariation information, they sometimes apply heuristics or knowledge and beliefs about potential mechanisms to assess causality instead (for a review see, for instance, Malle, 2004). Yet our research was not meant to determine when people use covariation information or to suggest that people predominantly or most of the time use covariation information. We merely tested if, when people do use covariation information, the assumptions underlying the covariation model are borne out.

\section{Deviations of rationality or functional information use?}

The covariation model is sometimes used as a normative model of human attribution (cf. White, 2002; Försterling \& Binser, 2002; Schimansky, Rössler, \& Haker 2012; Schuster, Ruble, \& Weinert, 1998). To the extent that normative logic dictates rational judgment, the phenomena we observed may be viewed as deviations of rationality. Yet, such a conclusion would be based on one particular and rather narrow view of rationality. Rather than viewing the observed phenomena as 'violations' of logic, let alone rationality, we would like to ask which social function they may serve.

It is possible, for instance, that differentially weighting different types of covariation information is effective from an approach-avoidance point of view. Another possibility is that the observed phenomena reflect real-life knowledge people accumulate during their lifetimes. If it is true that patients whom many actors treat in similar manners have generally caused this treatment themselves, there is no such thing like a 'violation' of whatever rule if observers focus on consensus rather than on 
distinctiveness when wondering about the patient's causal role. Within our Gricean framework, however, it is even more plausible that the covariation information that people infer from verbal communications reflects the covariation information that the communicator wished to convey. By saying that Peter kissed Mary, communicators may indeed share the insight that such is worth mentioning - and hence, that it is a rather remarkable event. Assuming that it says something specific about either the actor or the patient - or both - may thus simply be an effective manner for the receivers of the message to take part in the communicator's knowledge.

\section{Conclusion}

While explaining interpersonal events, people seem to sometimes use consensus and distinctiveness information interdependently, asymmetrically, and depending on whose causal role they try to assess. This is particularly true when they explain interpersonal events about which they are informed by verbal communication. It is predicted by a Gricean analysis of the conversational context in which people exchange behavioral information. Future research in the domain of causal attribution might therefore profit from taking this conversational context into account. 


\section{References}

Alicke, M. D., \& Insko, C. A. (1984). Sampling of similar and dissimilar comparison persons and objects as a function of the generality of attribution goal. Journal of Personality and Social Psychology, 46, 763-777.

Aronson, E. (2012). The Social Animal. New York: Worth/Freeman.

Bassili, J. N., \& Regan, D. T. (1977). Attributional focus as a determinant of information selection. The Journal of Social Psychology, 101, 113-121.

Bowler, M.C., Woehr, D.J., Bowler, J.L., Wuensch, K.L., \& McIntyre, M.D. (2011). The impact of interpersonal aggression on performance attributions. Group \& Organization Management, 36, 427-465.

Bruce, R. O., Cunningham, J. D., \& Kelley, H. H. (1975). A closer examination of causal inference: The roles of consensus, distinctiveness, and consistency information. Journal of Personality and Social Psychology, 32, 605-616.

Cheng, P.W. (1997). From covariation to causation: a causal power theory. Psychological Bulletin, 104, 367-405.

Coombs, W.T. (2007). Attribution theory as a guide for post-crisis communication research. Public Relations Review, 33, 135-139.

DiVitto, B., \& McArthur, L. Z. (1978). Developmental differences in the use of distinctiveness, consensus, and consistency information for making causal attribution. Developmental Psychology, 14, 474-482.

Eberly, M.B., Halley, E.C., Johnson, M.D., \& Mitchell, T.R. (2011). Beyond internal and external: A dyadic theory of relational attributions. Academy of Management Review, 36, 731-753. 
Fiske, S. T., Cuddy, A. J., \& Glick, P. (2007). Universal dimensions of social cognition: Warmth and competence. Trends in Cognitive Sciences, 11, 77-83.

Försterling, F. (1989). Models of covariation and attribution: How do they relate to the analogy of analysis of variance? Journal of Personality and Social Psychology, 57, 615-625.

Försterling, F., \& Binser, M.J. (2002). Depression, school performance, and the veridicality of perceived grades and causal attributions. Personality and Social Psychology Bulletin, 28, 1441-1449.

Garland, H., Hardy, A, \& Stephenson, L. (1975). Information search as affected by attribution type and response category. Personality and Social Psychology Bulletin, 1, 612-615.

Green, S.G., \& Mitchell, T.R. (1979). Attributional processes of leaders in leadermember interactions. Organizational Behavior and Human Performance, 23, $429-458$.

Greenwald, A.G. (2012). There is nothing so theoretical as a good method. Perspectives on Psychological Science, 7, 99-108.

Grice, H.P. (1975). Logic and conversation. In P. Cole \& J.L. Morgan (Eds.). Syntax and semantics 3: Speech acts (p. 41-58). San Diego: Academic Press.

Hansen, R. D. (1980). Commonsense attribution. Journal of Personality and Social Psychology, 39, 996-1009.

Hansen, R.D., \& Donoghue, J.M. (1977). The power of consensus: Information derived from one's own behavior. Journal of Personality and Social Psychology, 34, 425-433. 
Harris, L.T., \& Fiske, S.T. (2008). The brooms in Phantasia: Neural correlates of anthropomorphing objects. Social Cognition, 26, 210-223.

Hewstone, M., \& Jaspars, J. (1987). Covariation and causal attribution: A logical model of the intuitive analysis of variance. Journal of Personality and Social Psychology, 53, 663-672.

Hilton, D. J. (1995). The social context of reasoning: Conversational inference and rational judgment. Psychological Bulletin, 118, 248.

Hilton, D. (2007). Causal explanation: From social perception to knowledge-based causal attribution. In A.W. Kruglanski \& E.T. Higgins (Eds.), Social psychology: Handbook of basis principles (pp. 232-253). New York: The Guildford Press.

Hilton, D.J., \& Jaspars, J.M.F. (1987). The explanation of occurrences and nonoccurrences: A test of the inductive logic model of causal attribution. British Journal of Social Psychology, 26, 189-201.

Hilton, D. J., \& Slugoski, B. R. (1986). Knowledge-based causal attribution: The anormal conditions focus model. Psychological Review, 93, 75-88.

Hilton, D.J., Smith, R.H., \& Alicke, M.D. (1988). Knowledge-based information acquisition: Norms and the functions of consensus information. Journal of Personality and Social Psychology, 55, 530-540.

Hilton, D.J., Smith, R.H., \& Kim, S.H. (1995). Processes of causal explanation and dispositional attribution. Journal of Personality and Social Psychology, 68, 377387. 
Hoorens, V., Maier, J., \& Maris, S. (2012). When words speak louder: The effect of verb abstraction and valence on inferences from interpersonal events. Social Cognition, 30, 253-288.

Hortacsu, N. (1987). Attribution focus and information selection in relation tochronological age. Child Development, 58, 225 - 233.

Iacobucci, D., \& McGill, A.L. (1990). Analysis of attribution data: Theory testing and effects estimation. Journal of Personality and Social Psychology, 59, 426 - 441.

Jaspars, J. (1983). The process of causal attribution in common sense. In M. Hewstone (Ed.), Attribution theory: social and functional extensions (pp. 28-44). Oxford: Blackwell.

Jaspars, J., Hewstone, M., \& Fincham, F.D. (1983). Attribution theory and research: The state of the art. In J. Jaspars, F.D. Fincham, \& M. Hewstone (Eds.), Attribution theory and research: Conceptual, developmental and social dimensions (pp. 3-36). London: Academic Press.

Jiang, J., Gretzel, U., \& Law, R. (2010). Do negative experiences always lead to dissatisfaction? Testing attribution theory in the context of online travel reviews. Information and Communication Technologies in Tourism, 8, 297-308.

Kassin, S. M. (1979). Consensus information, prediction, and causal attribution: A review of the literature and issues. Journal of Personality and Social Psychology, 37, 1966-1981.

Kelley, H. H. (1967). Attribution theory in social psychology. In D. Levine (Ed.). Nesbraska symposium on motivation. Volume 15 (pp. 129-238). Lincoln: University of Nebraska Press. 
Kelley, H.H. (1973). The process of causal attribution. American Psychologist, 107, $107-128$.

Klein, K.M., Apple, K.J., \& Kahn, A.S. (2011). Attributions of blame and responsibility in sexual harassment: Reexamining a psychological model. Law \& Human Behavior, 35, 92-103.

Koonce, L., Seybert, N., \& Smith, J. (2011). Causal reasoning in financial reporting and voluntary disclosure. Accounting, Organization and Society, 36, 209-225.

Lalljee, M., Lamb, R., Furnham, A., \& Jaspars, J. (1984). Explanations and information search: Inductive and hypothesis-testing approaches to arriving at an explanation. British Journal of Social Psychology, 23, 201-212.

Major, B. (1980). Information acquisition and attribution processes. Journal of personality and Social Psychology, 39, 1010-1023.

Malle, B.F. (2004). How the mind explains behavior. Folk explanations, meaning, and social interaction. Cambridge: The MIT Press. CHECK OF IK DAT NOG

\section{GEBRUIK}

Martinko, M.J., \& Thomson, N.F. (1997). A synthesis and extension of the Weiner and Kelley attribution models. Basic and Applied Social Psychology, 20, 271-284.

McArthur, L. A. (1972). The how and what of why: Some determinants and consequences of causal attribution. Journal of Personality and Social Psychology, 22, 171-193.

McArthur, L. A. (1976). The lesser influence of consensus than distinctiveness information on causal attributions: A test of the person-thing hypothesis. Journal of Personality and Social Psychology, 33, 733-742. 
McArthur, L. Z. (1980). Illusory causation and illusory correlation :Two epistemological accounts. Personality and Social Psychology Bulletin, 6, 507519.

McClure, J, Allen, M.W., \& Wolkey, F. (2001). Countering fatalism: Causal information in news reports affects judgments about earthquake damage. Basic and Applied Social Psychology, 23, 109-121.

McKenzie, C.R.M. \& Mikkelsen, L.A. (2007). A Bayesian view of covariation assessment. Cognitive Psychology, 54, 33-61.

McKenzie, C.R.M., Ferreira, V.S., Mikkelsen, L.A., McDermott, K.J., \& Skrable, R.P. (2001). Do conditional hypotheses target rare events? Organizational Behavior and Human Decision Processes, 85(2), 291-309.

Miller, F. D., Smith, E. R., \& Uleman, J. (1981). Measurement and interpretation of situational and dispositional attributions. Journal of Experimental Social Psychology, 17, 80-95.

Nickerson, R.S. (1998). Confirmation bias: A ubiquitous phenomenon in many guises. British Journal of General Psychology, 2, 175-220.

Oaksford, M. \& Chater, N. (1994). A rational analysis of the selection task as optimal data selection. Psychological Review, 101, 608-631.

Orvis, B.R., Cunningham, J.D., \& Kelley, H.H. (1975). A closer examination of causal inference: The roles of consensus, distinctiveness, and consistency information. Journal of Personality and Social Psychology, 32, 605-616.

Pandelaere, M. Hoorens, V., Peeters, G. (2003). Why ask about Peter? Do you think he caused it? How the description of causal events guides the selection of questions about them? Basic and Applied Social Psychology, 25, 291-297. 
Perales, J.C., \& Shanks, D.R. (2003). Normative and descriptive accounts of the influence of power and contingency on causal judgment. The Quarterly Journal of Experimental Psychology, 56A, 977-1007.

Ployhart, R.E., Ehrhart, K.H., \& Hayes, S.C. (2005). Using attributions to understand the effects of explanations on applicant reactions: Are reactions consistent with the covariation principle? Journal of Applied Social Psychology, 35, 259-296.

Quayle, M., \& Naidoo, E. (2012). Social risk and attribution: how considering the social risk of attributions can improve the performance of Kelley's ANOVA model in applied research. Journal of Applied Social Psychology, 42, 1694-1715.

Rees, T., Ingledew, D.K., \& Hardy, L. (2005). Attribution in sport psychology: Seeking congruence between theory, research and practice. Psychology of Sports and Exercise, 6, 189-204.

Rose, J.P., Windschitl, P.D., \& Jenson, M.E. (2011). The joint influence of consensus information and situational information on trait inferences for targets and populations. Social Cognition, 29, 147-165.

Ruble, D. N., \& Feldman, N. S. (1976). Order of consensus, distinctiveness, and consistency information and causal attribution. Journal of Personality and Social Psychology, 34, 930-937.

Rudolph, U., \& Försterling, F. (1997). The psychological causality implicit in verbs: A review. Psychological Bulletin, 121, 192-218.

Rudolph, U., \& von Hecker, U. (2006). Three principles of explanation. Verb schemas, balance, and imbalance repair. Journal of Language and Social Psychology, 25, 377-405. 
Ryan, J., \& Boscia, M.W. (2003). Using attribution theory to help frame moral dilemma's: An empirical test of the president Clinton-Monica Lewinsky case. Teaching Business Ethics, 7, 123-137.

Samnani, A.K., Singh, P., \& Ezzedeen, S. (2013). Workplace bullying and employee performance: An attributional model. Organizational Psychology Review, 3, 337-359.

Schimansky, J., Rössler, W., \& Haker, H. (2012). The influence of social cognition on ego disturbances in patients with Schizophrenia. Psychopathology, 45, 117-125.

Schuster, B., Ruble, D.N., \& Weinert, F.E. (1998). Causal inferences and the positivity bias in children: The role of the covariation principle. Child Development, 69, 1577-1596.

Schwarz, A. (2008). Covariation-based causal attributions during organizational crises: Suggestions for extending Situational Crisis Communication Theory (SCCT). International Journal of Strategic Communication, 2, 31-53.

Schwarz, A. (2012). Stakeholder attributions in crises: The effects of covariation information and attributional inferences on organizational reputation. International Journal of Strategic Communication, 6, 174-195.

Schwarz, N., Strack, F., Hilton, D., \& Naderer, G. (1991). Base rates, representativeness, and the logic of conversation: The contextual relevance of “irrelevant” information. Social Cognition, 9, 67-84.

Seiver, E., Gopnik, A., \& Goodman, N.D. (2013). Did she jump because she was the big sister or because the trampoline was safe? Causal inference and the development of social attribution. Child Development, 84, 443-454. 
Semin, G. R., \& Fielder, K. (1988). The cognitive functions of linguistic categories in describing persons: social cognition and language. Journal of Personality and Social Psychology, 54, 558-568.

Solomon, S. (1978). Measuring dispositional and situational attributions. Personality and Social Psychology Bulletin, 4, 589-594.

Strack, F., Schwarz, N., \& Wänke, M. (1991). Semantic and pragmatic aspects of context effects in social and psychological research. Social Cognition, 9, 111125.

Sutton, R.M., \& McClure, J. (2001). Covariational influences on goal-based explanation: An integrative model. Journal of Personality and Social Psychology, 80, 222-236.

Van Overwalle, F. (1997). A test of the joint model of causal attribution. European Journal of Social Psychology, 27, 221-236.

Van Overwalle, F. (2003). Acquisition of dispositional attributions: effects of sample size and covariation. European Journal of Social Psychology, 33, 515-533.

Wegner, D. M., Wenzlaff, R., Kerker, R. M., \& Beattie, A. E. (1981). Incrimination through innuendo: Can media questions become public answers? Journal of Personality and Social Psychology, 40, 822.

Weiner, B. (2008). Reflections of the history of attribution theory and research. Social Psychology, 39, 151-156.

Wells, G. L., \& Harvey, J. H. (1977). Do people use consensus information in making causal attribution? Journal of Personality and Social Psychology, 35, 279-293.

White, P.A. (2002). Causal attribution from covariation information: the evidential evaluation model. European Journal of Social Psychology, 32, 667-684. 
White, P.A. (2003a). Causal judgement as evaluation of evidence: The use of confirmatory and disconfirmatory evidence. The Quarterly Journal of Experimental Psychology, 56, 491-513.

White, P. A. (2003b). Making causal judgments from the proportion of confirming instances: The pCI rule. Journal of Experimental Psychology: Learning, Memory, and Cognition, 29, 710-727.

Wojciszke, B., \& Abele, A.E. (2008). The primacy of communion over agency and its reversals in evaluations. European Journal of Social Psychology, 38, 1139-1147.

Wright, E. F., Lüüs, C. A. E., \& Christie, S. D. (1990). Does group discussion facilitate the use of consensus information in making causal attributions? Journal of Personality and Social Psychology, 59, 261-269. 
Table 1 Mean Causality Ratings As A Function of Interactant, Consensus,

Distinctiveness, and Consistency

\begin{tabular}{lcccccc}
\hline \hline & \multicolumn{3}{c}{ Actor } & \multicolumn{3}{c}{ Patient } \\
\cline { 2 - 6 } Distinctiveness & High & No & Low & High & No & Low \\
& Consensus & Consensus & Consensus & Consensus & Consensus & Consensus \\
\hline
\end{tabular}

\section{Study 1}

\begin{tabular}{lllll} 
High & 5.1 & 5.9 & 6.4 & 5.2 \\
Low & 6.7 & 7.1 & 5.9 & 3.9 \\
\hline
\end{tabular}

Study 2

\begin{tabular}{lllllll} 
High & 4.9 & 5.4 & 6.3 & 6.8 & 6.4 & 6.8 \\
No & 4.8 & 6.3 & 7.4 & 6.8 & 5.2 & 3.9 \\
Low & 6.6 & 7.5 & 7.3 & 6.2 & 3.1 & 3.6 \\
\hline
\end{tabular}

Study 3

\begin{tabular}{lllllll} 
& \multicolumn{5}{c}{ Low consistency } \\
High & 6.1 & 6.4 & 6.5 & 5.2 & 4.7 & 4.3 \\
No & 6.2 & 6.4 & 6.5 & 5.1 & 4.5 & 4.1 \\
Low & 6.8 & 7.0 & 7.0 & 4.8 & 4.0 & 3.8
\end{tabular}

\begin{tabular}{lllllll} 
& \multicolumn{5}{c}{ No consistency } \\
High & 6.2 & 6.5 & 6.8 & 5.3 & 5.1 & 4.6 \\
No & 5.8 & 6.9 & 7.3 & 5.6 & 4.0 & 3.7 \\
Low & 6.9 & 7.5 & 7.4 & 4.9 & 3.0 & 3.6
\end{tabular}

High consistency

\begin{tabular}{lllllll} 
High & 6.5 & 6.8 & 6.8 & 5.2 & 4.7 & 4.7 \\
No & 6.3 & 7.1 & 7.3 & 5.5 & 4.3 & 3.8 \\
Low & 6.9 & 7.4 & 7.5 & 5.0 & 3.5 & 3.4 \\
\hline
\end{tabular}




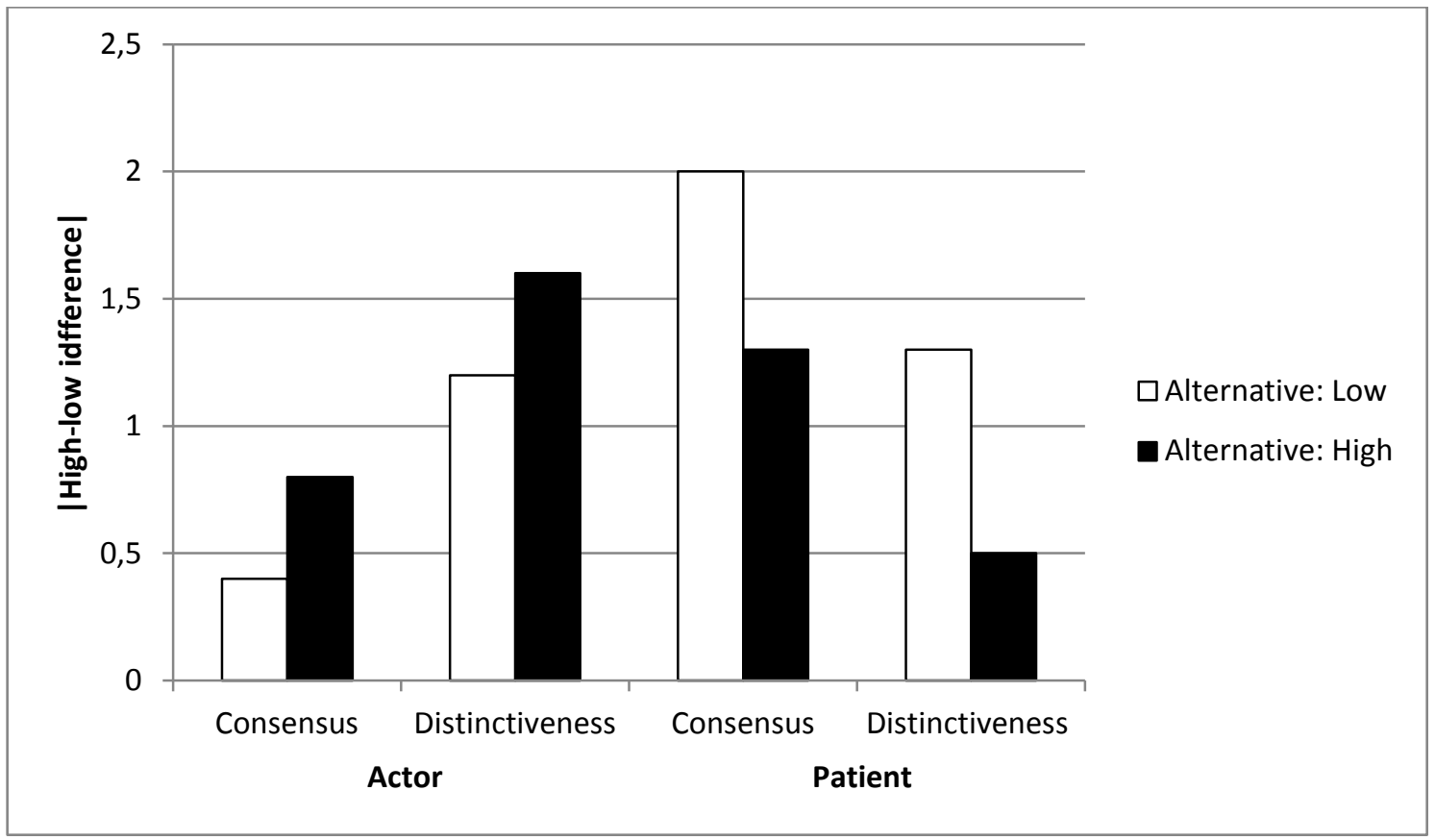

Figure 1. The effect of consensus and distinctiveness information (expressed as the absolute magnitude of the high-low difference) on actor and patient causality ratings as a function of the information on alternative dimension (Study 1). 